\title{
Correction to: Intratype variants of the E2 protein from human papillomavirus type 18 induce different gene expression profiles associated with apoptosis and cell proliferation
}

\author{
Alma Mariana Fuentes-González ${ }^{1,2}$. J. Omar Muñoz-Bello ${ }^{1,2} \cdot$ Joaquín Manzo-Merino $^{3}$ Adriana Contreras-Paredes $^{2}$. \\ Abraham Pedroza-Torres $^{3}$ - Jorge Fernández-Retana ${ }^{2} \cdot$ Carlos Pérez-Plasencia $^{2} \cdot$ Marcela Lizano $^{2,4}$ (D)
}

Published online: 18 May 2019

(c) Springer-Verlag GmbH Austria, part of Springer Nature 2019

\section{Correction to: Archives of Virology https://doi.org/10.1007/s00705-018-04124-6}

The Given names of the author Alma Mariana FuentesGonzález was incorrectly tagged in original publication and corrected here. The original article has been corrected.

Publisher's Note Springer Nature remains neutral with regard to jurisdictional claims in published maps and institutional affiliations.

The original article can be found online at https://doi.org/10.1007/ s00705-018-04124-6.

Marcela Lizano

lizanosoberon@gmail.com

Alma Mariana Fuentes-González

alma_mariana2000@yahoo.com.mx

J. Omar Muñoz-Bello

omarmube@gmail.com

Joaquín Manzo-Merino jmanzomerino@gmail.com

Adriana Contreras-Paredes adrycont@yahoo.com.mx

Abraham Pedroza-Torres abraneet@gmail.com

Jorge Fernández-Retana fernandezretana@gmail.com

Carlos Pérez-Plasencia carlos.pplas@gmail.com
1 Programa de Doctorado en Ciencias Biomédicas, Universidad Nacional Autónoma de México, Ciudad Universitaria, 04510 Mexico City, Mexico

2 Unidad de Investigación Biomédica en Cáncer, Instituto Nacional de Cancerología, México/Instituto de Investigaciones Biomédicas, Universidad Nacional Autónoma de México, Av. San Fernando No. 22, Col. Sección XVI, Tlalpan, 14080 Mexico City, Mexico

3 CONACyT-Instituto Nacional de Cancerología, Av. San Fernando No. 22, Col. Sección XVI, Tlalpan, 14080 Mexico City, Mexico

4 Departamento de Medicina Genómica y Toxicología Ambiental, Instituto de Investigaciones Biomédicas, Universidad Nacional Autónoma de México, 04510 Mexico City, Mexico 